\title{
The close T Tauri binary V 4046 Sagittarii ${ }^{\star}$
}

\author{
H. C. Stempels ${ }^{1, \star \star}$ and G. F. Gahm ${ }^{2}$ \\ 1 Department of Astronomy and Space Physics, Box 515, 75120 Uppsala, Sweden \\ 2 Stockholm Observatory, AlbaNova University Center, 10691 Stockholm, Sweden
}

Received 13 October 2003 / Accepted 1 April 2004

\begin{abstract}
We have collected high-resolution $(R \approx 60000)$ VLT-UVES spectrograms of the close T Tauri binary V $4046 \mathrm{Sgr}$ from $3500-6750 \AA$ at different phases of its 2.4 day orbital period. The high quality of these spectra allows us to present an improved ephemeris of the system. To model the photospheric absorption line spectrum we calculate synthetic spectra for the observed phases of the system. These synthetic spectra are used to determine veiling levels, and to extract emission line profiles that are undistorted by photospheric absorption lines. We find that the shapes of the strong emission lines of $\mathrm{H}$ and $\mathrm{Ca}$ II $\mathrm{H} \& \mathrm{~K}$ all vary periodically with phase. A weak veiling continuum is superimposed on the stellar absorption line spectra. The Ca II H \& K emission lines are composed of two narrow emission components that closely follow the stellar orbital motion, and we speculate that these lines are formed in global chromospheric networks on the stars. Also the Balmer lines have similar narrow "stellar" components, possibly also chromospheric. However, in addition there are extended wings on each side of the line centers of all $\mathrm{H}$ lines, which change dramatically in shape with the orbital phase. We find that the shape and velocity changes of the wing components are consistent with two concentrations of gas moving at high velocity but co-rotating with the stars. These concentrations move with a projected velocity of $80 \mathrm{~km} \mathrm{~s}^{-1}$ around the center of mass. They are located well inside the edge of the circumbinary disk, and also inside the co-linear Lagrangian points of this binary. With this concept we obtain a very good agreement between calculated and observed line profiles of H8, H9 and H10 as a function of phase. In some recent calculations of mass transfer from circumbinary disks to close binaries in circular motion, accumulations of gas in co-rotation with the stars develop, resulting in structures which are similar to what we have found from our observations. We also investigate the cause of periodic photometric variations observed by others.
\end{abstract}

Key words. stars: pre-main sequence - stars: binaries (including multiple): close - accretion, accretion disks

\section{Introduction}

$\mathrm{T}$ Tauri stars are identified with fully convective, contracting pre-main-sequence objects of solar mass and thereabouts. A fraction of these stars, the classical T Tauri stars (CTTSs), have prominent and broad emission lines in the optical spectral region. Since these stars are also surrounded by rather thick, dusty disks it has been proposed that the strong line emission is related to an interaction between disk and star. In particular, models involving accretion of matter from the disk to the star along stellar dipole magnetic fields have successfully accounted for many of the peculiarities observed in CTTSs. The observations and the theory of magnetospheric accretion was summarized in the book by Hartmann (1998), and has developed since.

Many T Tauri stars are double or multiple. Most of the theoretical work so far concerns single stars but should also be

Send offprint requests to: $\mathrm{H}$. C. Stempels,

e-mail: Eric.Stempels@not.iac.es

* Based on observations collected at the European Southern Observatory, Chile (proposal 69.C-0481).

$\star \star$ Present address: Nordic Optical Telescope, Apartado 474, 38700 Santa Cruz de La Palma, Spain. applicable to the individual components in wide pairs, since each component may carry a disk of its own. The closer pairs, however, may create a central gap in what becomes a circumbinary disk. Such disks may form even for separations between the components of several tens of AU. An example is GG Tau with a projected separation of 40 AU (Silber et al. 2000). For reviews of young binary stars, see for example Mathieu (1994) and Mathieu et al. (2000).

Of the discovered close binaries, most are so called weaklined T Tauri stars, with little or no evidence of interaction between star and disk. Only a few CTTS binaries with separations smaller than $1 \mathrm{AU}$ are known: GW Ori $(P=242 \mathrm{~d}$, Mathieu et al. 1991), RX J0530.7-0434 ( $P=40.6 \mathrm{~d}$, Covino et al. 2001), UZ Tau E $(P=19.1 \mathrm{~d}$, Mathieu et al. 1996), DQ Tau ( $P=15.8 \mathrm{~d}$, Mathieu et al. 1997) and V $4046 \mathrm{Sgr}$ $(P=2.4 \mathrm{~d}$, Byrne 1986). All have emission line signatures characteristic of single CTTSs, as if the process of accretion operates equally well in the case of binary components inside a circumbinary disk. For DQ Tau Basri et al. (1997) found periodic bursts of emission, pointing at enhanced mass accretion at periastron passages. Such bursts can be expected for components in elliptical orbits simply from gravitational perturbations of the disk (Artymowicz \& Lubow 1996). Also RX J0530.7 
and UZ Tau E have rather elliptical orbits. For the latter Prato et al. (2002) found that the disk and binary orbits are probably coplanar.

The photospheric spectra of CTTSs are distorted by superimposed emission lines and veiling, and one may suspect that more very close pairs are still to be revealed. RW Aur A is a suspected close binary (Gahm et al. 1999; Petrov et al. 2001), like AA Tau (Bouvier et al. 2003). Also RU Lup (Takami et al. 2001) and RY Lup (Gahm et al. 1989) have been considered, but no conclusive evidence has been found.

We have made a high-resolution spectroscopic study of one of the objects listed above, namely V 4046 Sgr (HDE 319139 , AS 292), a double-lined spectroscopic binary with $V=10.5^{\mathrm{m}}$. Quast et al. (2000) estimated a distance of $83 \mathrm{pc}$ to the system. They also derived a period of $2.4213305 \pm 0.000004$ days, and masses of $0.86 M_{\odot}$ and $0.69 M_{\odot}$ for the components of spectral types K5Ve and K7Ve. Rapid changes in the strength and line profiles of $\mathrm{H} \alpha$ with phase were reported by Byrne (1986), de la Reza et al. (1986), Quast et al. (2000) and Mekkaden (2000). V 4046 Sgr is an isolated T Tauri star, but besides the time-dependent features it is a typical CTTS, also in the far-UV, where de la Reza et al. (1986) observed changes in the line emission fluxes. Like many other CTTSs it has been reported to flare in the photometric $U$ band (Busko \& Torres 1976, 1978).

The infrared energy distribution of V 4046 Sgr suggests that V 4046 Sgr has cleared a gap in a circumbinary disk (Jensen \& Mathieu 1997). In this gap the components move in nearly circular orbits, and the system was included by Melo et al. (2001) in a discussion of timescales for circularization of orbits. Up to now, a system with circular orbits was not expected to induce much mass accretion from gravitational perturbations of its disk. However, our investigation shows that complex gas motions exist in V 4046 Sgr. The focus of the present paper lies on the mapping and interpretation of these gas flows.

\section{Observations and orbital parameters}

The orbital period of V $4046 \mathrm{Sgr}$ is $\sim 2.4$ days. Therefore 4 consecutive nights of observations are just sufficient to obtain a reasonable coverage of the orbital phases. During the nights of April 15-18, 2002, we obtained 8 high-quality spectra of V 4046 Sgr with the UVES spectrograph and the 8-m VLT at Cerro Paranal, Chile. The UVES/VLT combination is a powerful instrument for investigating time-dependent processes in T Tauri stars because of its ability to combine a high spectral resolution $(R>60000)$ with a large wavelength range (3500-6750 $\AA$ in our setup) and a high signal-to-noise ratio. An exposure time of $15 \mathrm{~min}$ provided us with spectra with a signal-to-noise ratio per resolution element of approximately 25 at the shortest wavelengths, increasing to 300 at the longest wavelengths.

The observed data were reduced with the package REDUCE (Piskunov \& Valenti 2002). This package, written in IDL, performs optimal order extraction through 2D modelling of the individual order shapes, as well as bias-subtraction

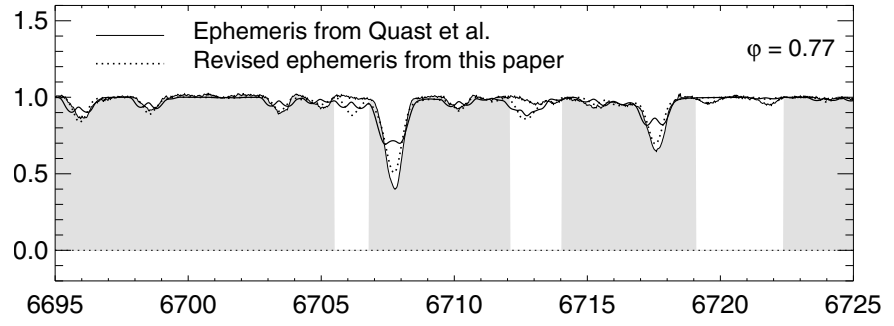

Fig. 1. In this figure we compare a synthetic spectrum based on the ephemeris of Quast et al. (thick line) and a synthetic spectrum based on the revised ephemeris presented in this paper (dotted line with the observed spectrum of the Li I 6707 absorption line (thin line). The mid-exposure JD of this spectrum is 2452 382.745. Details about the calculation of synthetic spectra are given in Sect. 3 .

and flat-fielding. The final spectra were shifted to the heliocentric reference frame.

\subsection{Ephemeris and orbital parameters}

We performed a standard cross-correlation analysis on the obtained spectra to obtain accurate radial velocities of the two components of the system. We then used the method of least-squares to determine the most likely values for the initial epoch $T_{0}$ and the period $P$, which gave the solution $T_{0}=$ $2452380.867 \pm 0.03$ (close to our first observation) and $P=$ $2.42537 \pm 0.01$ days. These values are close to the ephemeris determined by Quast et al. (2000) $\left(T_{0}=2446998.335 \pm 0.002\right.$ and $P=2.4213305 \pm 0.000004$ days), but, after correction for the phase offset of 0.0514 due to the change in distance to the system between the two different $T_{0}$ 's, we find that there still is a small but significant discrepancy when we apply the ephemeris of Quast et al. to our data, as illustrated in Fig. 1. The difference between our solution and that of Quast et al. corresponds to an offset of 0.034 in phase, or about $50 \mathrm{~min}$ in time. Therefore, to obtain the best agreement with our observations, as well as with the ephemeris of Quast et al., we decided to use the following ephemeris in our analysis of the spectra:

$\mathrm{JD}=2446998.335+2.4213459 \varphi$.

From our cross-correlation analysis we also found that the amplitudes of the radial velocities of the components are $K_{\text {pri }}=$ 54.16 and $K_{\mathrm{sec}}=56.61$. With an assumed inclination $i$ of $35^{\circ}$, this gives dynamical masses of the components of $M_{\text {pri }}=$ $0.912 M_{\odot}$ and $M_{\mathrm{sec}}=0.873 M_{\odot}$. These velocity amplitudes and masses are slightly larger than the values given by Quast et al. On the other hand, they also noted a problem when comparing dynamical masses with model predictions, but we can conclude that both our revised masses and the mass ratio are in satisfactory agreement with recent pre-main-sequence models described by Palla \& Stahler (1999) and Baraffe et al. (1998). Also the derived ages for the components are practically equal, about $10^{7}$ years.

In Fig. 2 we show schematically what the geometry of this binary system may look like. In this figure the disk starts at $1.8\left(a_{1}+a_{2}\right)$ from the center of mass, as assumed for a dynamically cleared disk. We note, however, that 

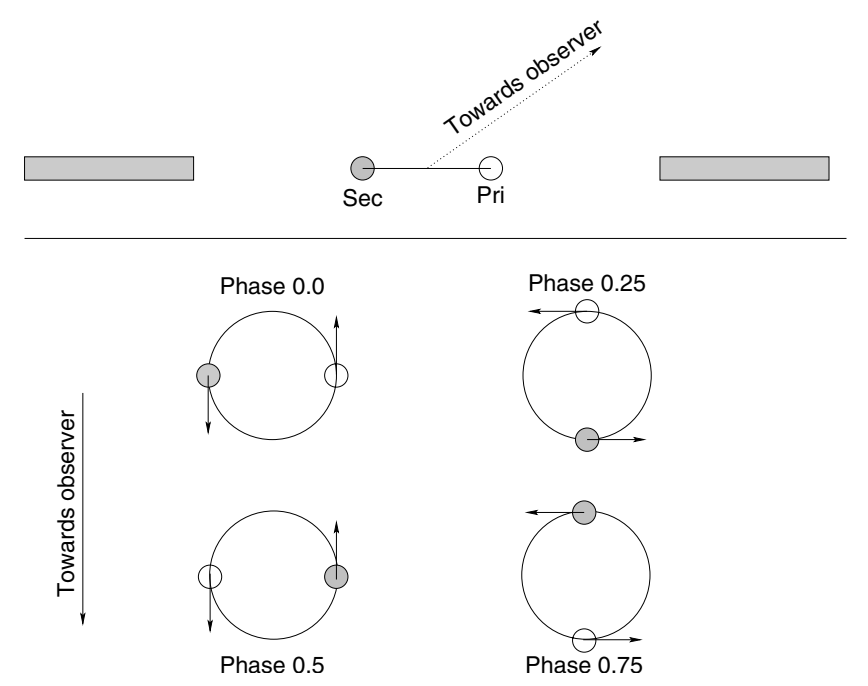

Fig. 2. Schematic presentation of the system. The upper figure illustrates an edge-on impression of the system. Distances and stellar radii are proportional. The inner boundary of the circumbinary disk is located at $1.8\left(a_{1}+a_{2}\right)$ from the center of mass (see text). The lower figures show the relative positions of the stars at selected phases.

Jensen \& Mathieu (1997) find evidence for a more extended gap from modeling the near-infrared excess of V $4046 \mathrm{Sgr}$.

We also analyzed the radial velocity variations published by Quast et al., and derived an ellipticity of the orbit of $e \leq 0.01$. We list the revised orbital parameters in Table 1.

\section{Spectral synthesis}

To analyze our observations in V 4046 Sgr we constructed synthetic spectra for each component using the package SME (Valenti \& Piskunov 1996). Synthetic spectra have been used successfully to describe the photospheres of T Tauri stars (Stempels \& Piskunov 2002, 2003), and the same method is used in this investigation.

For each component of V 4046 Sgr we calculated synthetic spectra using the latest generation of MARCS model atmospheres (Gustafsson et al. 2002). Spectral line data were obtained from the Vienna Atomic Line Database (Piskunov et al. 1995; Kupka et al. 1999; Ryabchikova et al. 1999). As a starting point for our calculations, we used the atmospheric parameters $T_{\text {eff }}, \log g, v \sin i, v_{\text {rad }}$ determined by Quast et al. (2000). Apart from the stellar contribution to the total flux, an additional contribution from a possible veiling continuum is not uncommon in CTTSs. For a reasonably short spectral interval, at least up to $100 \AA$, the contribution of the veiling continuum can be modelled as a featureless continuum that reduces the depth of the spectral lines. Such a model is included in our spectral synthesis calculations.

An advantage of using synthetic spectra for modelling the stellar photosphere is that radiative transfer calculations provide the absolute flux levels in the spectral lines and the continuum regions of the object. This makes it possible to determine the true level of the continuum, even in the case of strong line blanketing. This is especially helpful in the bluest part of the spectrum, because in these regions continuum definition from
Table 1. Orbital parameters of V 4046 Sgr.

\begin{tabular}{llc}
\hline \hline Parameter & Primary & Secondary \\
\hline$K$ & $54.16 \mathrm{~km} \mathrm{~s}^{-1}$ & $56.61 \mathrm{~km} \mathrm{~s}^{-1}$ \\
$M$ & $0.912 M_{\odot}$ & $0.873 M_{\odot}$ \\
$a_{1}, a_{2}$ & $4.52 R_{\odot}$ & $4.72 R_{\odot}$ \\
$P$ & \multicolumn{2}{c}{$2.4213459 \mathrm{~d}$} \\
$i^{1}$ & \multicolumn{2}{c}{$35^{\circ}$} \\
$e$ & $\leq 0.01$ \\
$v_{\text {rad }}{ }^{1}$ & \multicolumn{2}{c}{$-6.94 \mathrm{~km} \mathrm{~s}^{-1}$} \\
\hline${ }^{1}$ From Quast et al. (2000). &
\end{tabular}

observations alone may be very difficult. Thus, if the continuum level with respect to the absorption line depth is known from spectral synthesis, and the observed absorption line profiles span a range of different depths, it is possible to solve for both the continuum level and a possible veiling contribution. In this way we are able to obtain very clean emission line spectra.

We adapted some routines of SME to produce a flux-weighted combination of synthetic spectra of the individual components of V $4046 \mathrm{Sgr}$ at an arbitrary phase $\varphi$. In this routine we adjust the wavelength scale of each component with $\lambda_{i}^{\prime}=\lambda\left(1+\left(V_{i} \cos \varphi\right) / c\right)$, where $V_{i}$ is the maximum radial velocity of the component and $c$ the speed of light. Then the combined spectrum $F_{1+2}$ can be expressed by the simple equation:

$F_{1+2}(\lambda)=\frac{F_{1}\left(\lambda_{1}^{\prime}\right)+F_{2}\left(\lambda_{2}^{\prime}\right)}{F_{1}^{\mathrm{c}}\left(\lambda_{1}^{\prime}\right)+F_{2}^{\mathrm{c}}\left(\lambda_{2}^{\prime}\right)}$

where $F_{1}$ and $F_{2}$ are the respective flux spectra and $F_{1}^{\mathrm{c}}$ and $F_{2}^{\mathrm{c}}$ the respective continuum fluxes of the components, all directly derived from the radiative transfer calculations of SME. It should be noted that this method is equivalent to calculating a weighted combination of normalized spectra, where the relative weights are $F_{1}^{\mathrm{c}} / F_{2}^{\mathrm{c}}$ for the primary and 1 for the secondary. The corresponding relative weights that we recovered by using a combination of absolute flux spectra range from approximately $1.7: 1$ in the bluest part of our spectral range, to about $1.5: 1$ in the reddest part.

Using the orbital solution of Table 1, we meticulously compared for each phase the observed absorption line shapes with our synthetic spectra in a small spectral region around $\lambda=6000 \AA$ in order to assess whether the values of the stellar parameters $\left(T_{\mathrm{eff}}, \log g\right.$ and $\left.v \sin i\right)$ as determined by Quast et al. (2000) can be used for spectral synthesis. This particular wavelength region is well exposed, and contains spectral lines with well-defined atomic parameters. Our comparison yielded that the assumed stellar parameters gave satisfactory results, and could not be improved much further.

The final parameters we used for synthetic spectrum calculations are listed in Table 2, including the adopted values of micro- and macroturbulent velocities $\left(v_{\text {mic }}\right.$ and $\left.v_{\text {mac }}\right)$.

In our analysis below we frequently use subtraction of synthetic spectra from observed profiles to obtain the true shape of emission lines, uncontaminated by photospheric absorption features. In Fig. 3 we illustrate this procedure. We also show two examples of the excellent agreement between the observed 
Table 2. Stellar parameters of V 4046 Sgr used for spectral synthesis.

\begin{tabular}{lll}
\hline \hline Parameter & Primary & Secondary \\
\hline$T_{\text {eff }}$ & $4370 \mathrm{~K}$ & $4100 \mathrm{~K}$ \\
$\log g$ & 4.0 & 4.0 \\
$v \sin i$ & $14.2 \mathrm{~km} \mathrm{~s}^{-1}$ & $13.7 \mathrm{~km} \mathrm{~s}^{-1}$ \\
$v_{\text {mic }}$ & $1.0 \mathrm{~km} \mathrm{~s}^{-1}$ & $1.0 \mathrm{~km} \mathrm{~s}^{-1}$ \\
$v_{\text {mac }}$ & $2.0 \mathrm{~km} \mathrm{~s}^{-1}$ & $2.0 \mathrm{~km} \mathrm{~s}^{-1}$ \\
\hline
\end{tabular}

and synthetic spectrum in Fig. 4, displaying an absorption line complex around $\mathrm{Ca}$ I 6163, as well as an extended region around Li I 6707.

\section{Results}

\subsection{The absorption line spectrum and veiling}

The two K-type spectra contributing to the integrated light from V 4046 Sgr are normal for moderately rotating dwarfs. The equivalent width of $\mathrm{Li}$ I 6707 is large, $E W(\mathrm{Li})=0.5 \pm 0.03 \AA$ in each star. From our synthetic spectra calculations we derived an abundance of $N(\mathrm{Li}) / N(\mathrm{H})$ of $-8.5 \pm 0.2$ which puts the system at the very earliest phase of pre-main-sequence stellar evolution (Duncan 1981). There are no traces of TiO absorption in our spectra at any phase. In addition, we find no evidence of "shell"-absorption, either in the strong emission lines of $\mathrm{H}$, or as components in strong absorption lines from the ground state, central or shifted. The veiling continuum of $\mathrm{V} 4046 \mathrm{Sgr}$ is relatively weak in the visual region of the spectrum. Because spectral synthesis of all observed phases of a binary spectrum is tedious, we only measured the contribution of veiling at wavelengths corresponding to the spectral regions containing investigated emission lines, mainly lines of $\mathrm{H}$. In the bluest part of the spectrum $(\lambda \sim 3500-4000 \AA)$ veiling contributes one third of the total observed flux. Veiling decreases towards longer wavelengths. In the red part of the spectrum $(\lambda \sim 6000-6700 \AA)$ the level of veiling is only about $5-15 \%$ of the total flux, and is variable with phase.

\subsection{Photometric variability, extinction and synchroneous rotation}

Photometric fluctuations following the orbital period were reported by Quast et al. (2000) for the Johnson $B$ band, and Mekkaden (2000) for the Strömgren $y$ and $b$ bands. The corresponding flux level variations were very small, at most $\sim 5 \%$ of the total flux. These variations peak at phase 0.0 , when the secondary is approaching the observer, and the primary receding. Because of this geometry, it is hard to attribute the variability to mutual heating of opposing stellar hemispheres. It is also difficult to envision a case where the stars are periodically occulted by circumstellar dust in the line-of-sight, since the system has a substantial tilt (see Fig. 2).

We investigated whether the photometric fluctuations can be caused by variations in the level of veiling or by flux variability of the Balmer lines. In the Strömgren $y$ band, the veiling is only $5 \%$ of the total flux, and we find no evidence of periodic changes. Furthermore, simultaneous changes in the

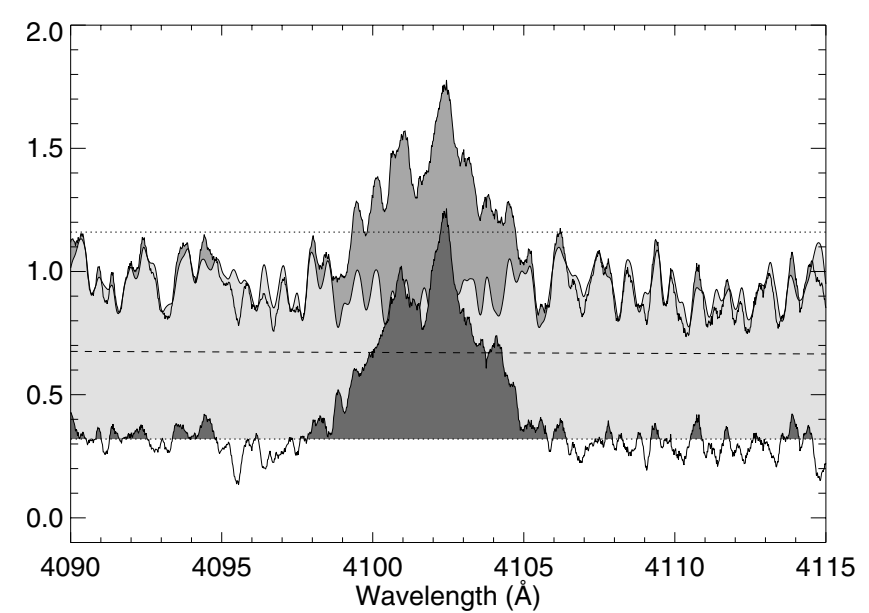

Fig. 3. This figure illustrates how the subtraction of a synthetic spectrum (light grey) from an observed spectrum (grey) eliminates the influence of stellar absorption lines on the emission line profile of $\mathrm{H} \delta$ (dark grey).

Balmer lines will contribute at most $1 \%$ in $B$. Therefore, we conclude that these emission components are not responsible for the fluctuations.

We note that Byrne (1986) did not find any evidence for periodic variations in $V$ or $B$. This may indicate that photometric variability is connected to activity on the star that comes and goes, and which for long periods may show dominant features, spots, on one side of the star(s). Synchroneous rotation (see below) will then cause this variablility to correlate with the orbital period. The observed color changes are small, and would not lead to any dramatic change in the observed spectral type. Also, the impact of a small number of starspots on the absorption line depth is very small, and we will not be able to detect such local changes in effective temperature from our spectral analysis.

Finally, from the average photometric colors derived in the photometric investigations cited above we can obtain a more precise value of the extinction to the star by correcting the colors for the color dependent veiling derived by us. We find practically no extinction, $A_{\mathrm{V}} \approx 0.0$, consistent with V 4046 Sgr being an isolated T Tauri star, and without substantial obscuration from foreground dust.

The flux ratios derived for this system are in agreement with the luminosity ratio derived by Quast et al. (2000). With the inferred sizes of the stars we agree with de la Reza et al. (1986) that both stars can be in synchronous rotation. Theoretical studies of close binary systems show that orbital synchronization occurs before circularization (Zahn 1977; Hilditch 2001). However, for pre-main sequence binaries close to the ZAMS, deviations from synchronization can occur due to accretion of circumstellar material (Zahn \& Bouchet 1989).

\subsection{Emission lines}

In our spectra of V $4046 \mathrm{Sgr}$ we detected strong emission from the Balmer lines ( $\mathrm{H} \alpha$ up to at least $\mathrm{H} 16$ ) and $\mathrm{Ca}$ II $\mathrm{H}$ and $\mathrm{K}$, as well as weak emission lines of He I 5876 and [O I] 6300. Furthermore, the Na I D lines are shallower than expected 

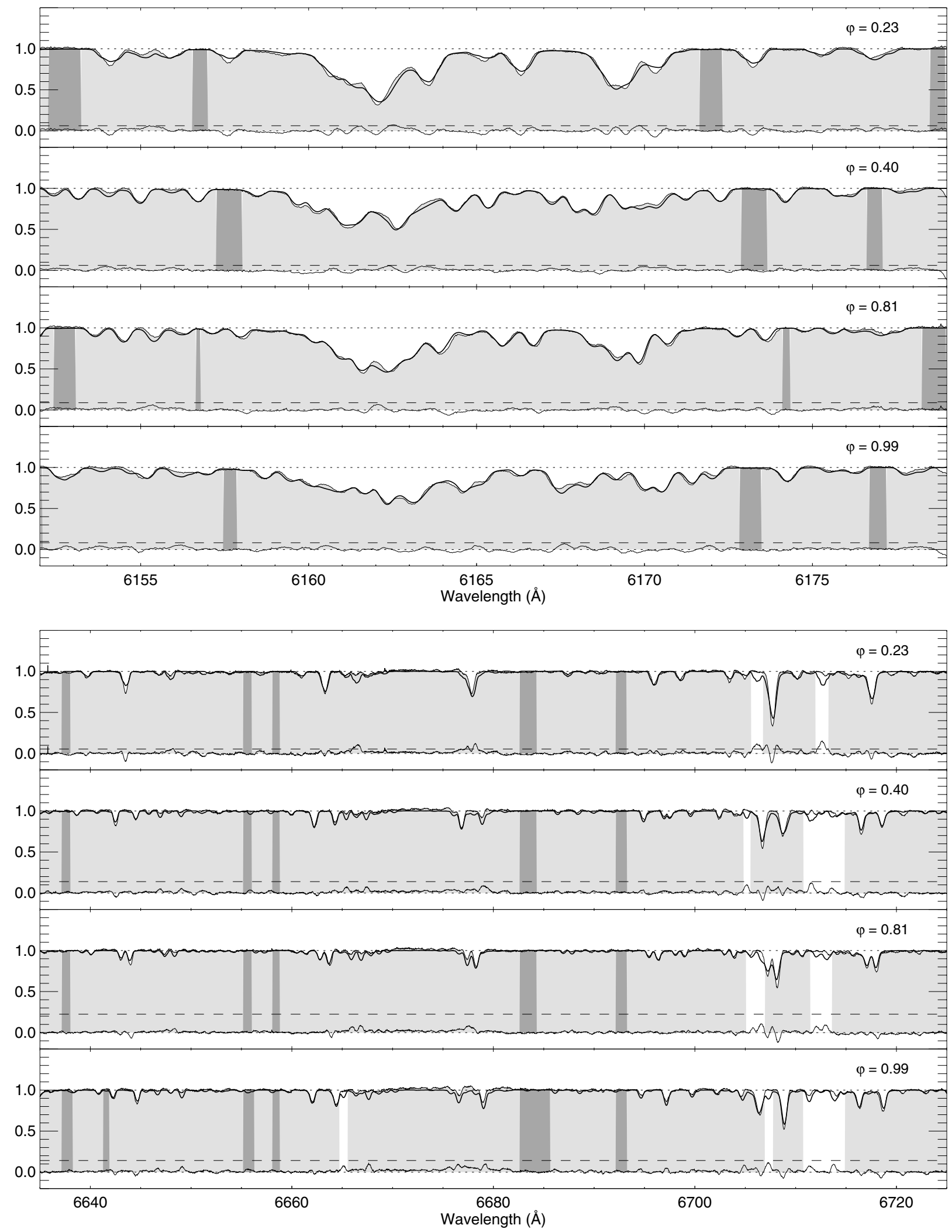

Fig. 4. The above two plots illustrates the good agreement between the observed and the synthetic spectrum at different phases. The observations are shown with a thin line, while the synthetic spectra are shown with a thick line. The synthetic spectrum includes veiling. The level of veiling is indicated by a horizontal dashed line. The thin line around $y=0$ is the difference between the observations and the synthetic spectrum. The dark grey areas indicate regions used for defining the continuum. The upper panel shows four phases of a complex of absorption lines near the Ca I 6162 line. The lower panel shows four phases of an extended region around the Li I 6707 line. One can also see a small amount of emission near the Fe I 6677.9 line. 

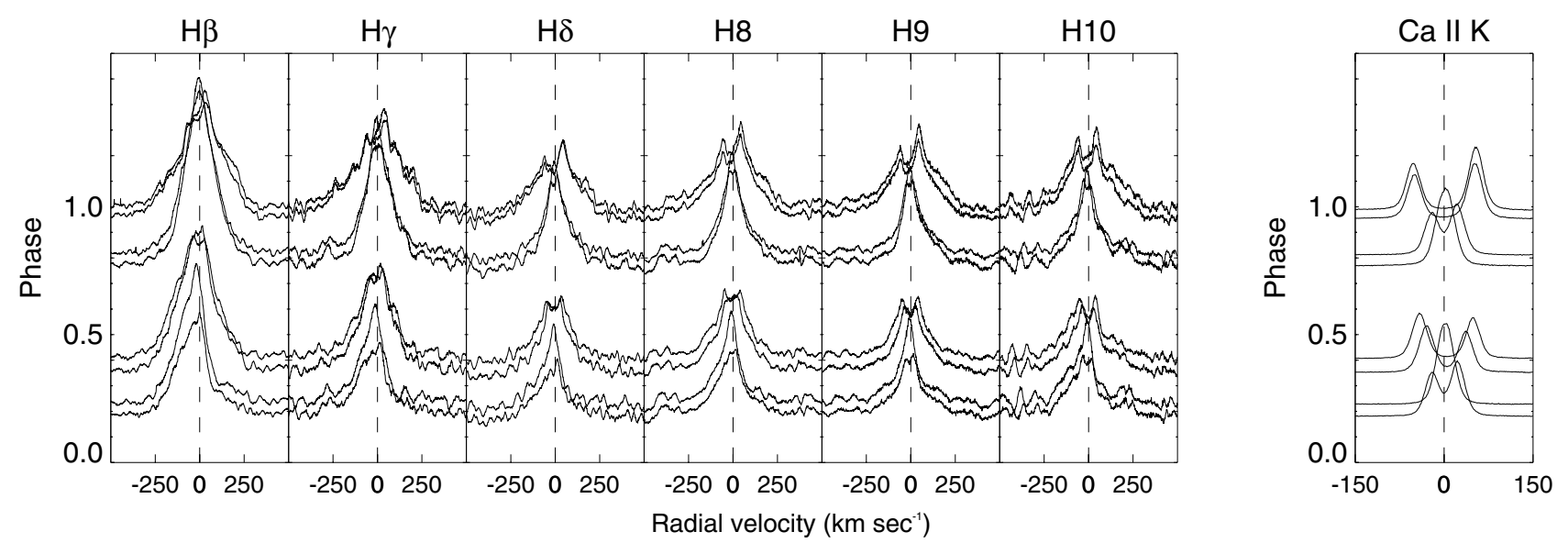

Fig. 5. This figure shows an overview of the strongest emission lines in V $4046 \mathrm{Sgr}$. A synthetic spectrum of the stellar contributions has been subtracted. The horizontal axes show the velocity in $\mathrm{km} \mathrm{s}^{-1}$ with respect to the center of mass velocity. The intensity of the profiles has been scaled to illustrate the line shapes rather than their relative intensities. The profiles are offset vertically according to orbital phase (see $y$-axis). The Ca II K line emission is very strong in comparison with the emission lines of $\mathrm{H}$, and therfore appears very smooth on this scale.

from our spectral synthesis, and probably partly filled in with emission. We derived the pure emission line spectrum by subtracting the synthetic spectrum of the stellar component and the veiling continuum. The emission line profiles we obtain in this way are much cleaner than the profiles including the stellar component. This also improves our measurements of the equivalent width of emission lines, because we are able to take into account all excess emission, not only emission that exceeds the continuum level.

We show an overview of the strongest emission lines in Fig. 5. $\mathrm{H} \alpha$ is excluded because it is so strong that it exceeded the linear regime of the CCD, and cannot be analyzed. $\mathrm{H} \epsilon$ is also excluded due to its blend with $\mathrm{Ca}$ II $\mathrm{H}$.

There are two $\mathrm{Ca}$ II $\mathrm{K}$ emission line components which are strongly coupled to each of the stellar components. Both components have almost exactly Gaussian profiles with a $F W H M=$ $23.5 \pm 0.5 \mathrm{~km} \mathrm{~s}^{-1}$, which is more than the stellar $v \sin i$ of 14.2 and $13.7 \mathrm{~km} \mathrm{~s}^{-1}$ for the primary and secondary, respectively. Still, the Ca II K lines follow closely the orbital motion of the binary. As seen in Fig. 5 the two components just add in flux at phases close to 0.25 and 0.75 , when the stars have zero radial velocity relative to the center of mass. Also, the strength and shape of the components do not vary with phase. We attribute each stellar component to global chromospheric emission from regions immediately surrounding the stars. Most of the line broadening is then due to rotational broadening of this extended chromosphere. Small-scale gas motions and turbulence in the chromosphere are likely to be present, producing a small additional line broadening. Finally, we note that the primary component is slightly stronger than the secondary component (a factor of $\sim 1.25$ ).

The Balmer lines are all strikingly similar in shape at a given phase, especially the higher members of the series. This similarity indicates that the lines have a common line formation region. However, in contrast to the Ca II $\mathrm{K}$ line, the shape and equivalent width of the Balmer lines vary dramatically as a function of phase. Also for these lines one can identify two stellar components with relatively narrow profiles, and it is likely that a substantial part of this emission originates in the global chromosphere discussed above. However, the Balmer lines are flanked with extended wings at all phases, as if in addition there are high velocity regions which are not directly connected to the stellar surface. We will discuss the Balmer lines in more detail in the subsequent section.

The equivalent widths of the Balmer lines and Ca II $\mathrm{K}$ are plotted against phase in Fig. 6. The behavior of the $E W$ s of the Balmer lines is very similar, being smallest close to phase 0.25 and largest around phase 1.0. We do not see any sinusoidal variation with phase. A similar tendency was found for $\mathrm{H} \alpha$ in previous investigations. These changes cannot be due to circumstellar obscuration, since then the star is expected to be occulted in the same way, leading to no change in $E W$. Also it is difficult to explain these variations with occultations by the disk or the stars at different phases. Earlier measurements show, however, a large scatter. Maybe there is an underlying pattern of periodic variability - a tendency - but disturbed by superimposed irregular flux variations, possibly due to real changes in the volume emission measures of all components.

Finally, we did not extract the residual emission in the $\mathrm{Na}$ I $\mathrm{D}$ lines because the exact profile of the extended absorption wings is uncertain. Also, for the weak He I 5876 and [O I] 6300 emission lines we can only state that the narrow components are there, just as for Ca II K. More highquality data could resolve if for instance He I also carries broad components.

\section{Analysis and modelling of hydrogen lines}

Quast et al. (2000) and Mekkaden (2000) reported a double period in the $F W H M$ of the $\mathrm{H} \alpha$ line, as well as periodic variations in the $\mathrm{H} \alpha$ peak intensity, but with a period of only half the orbital period. From Fig. 5 one can see that both these effects are consequences of how the two narrow, stellar components vary with phase. We can conclude that the reported periods result from orbital motion of the hydrogen emitting regions rather 


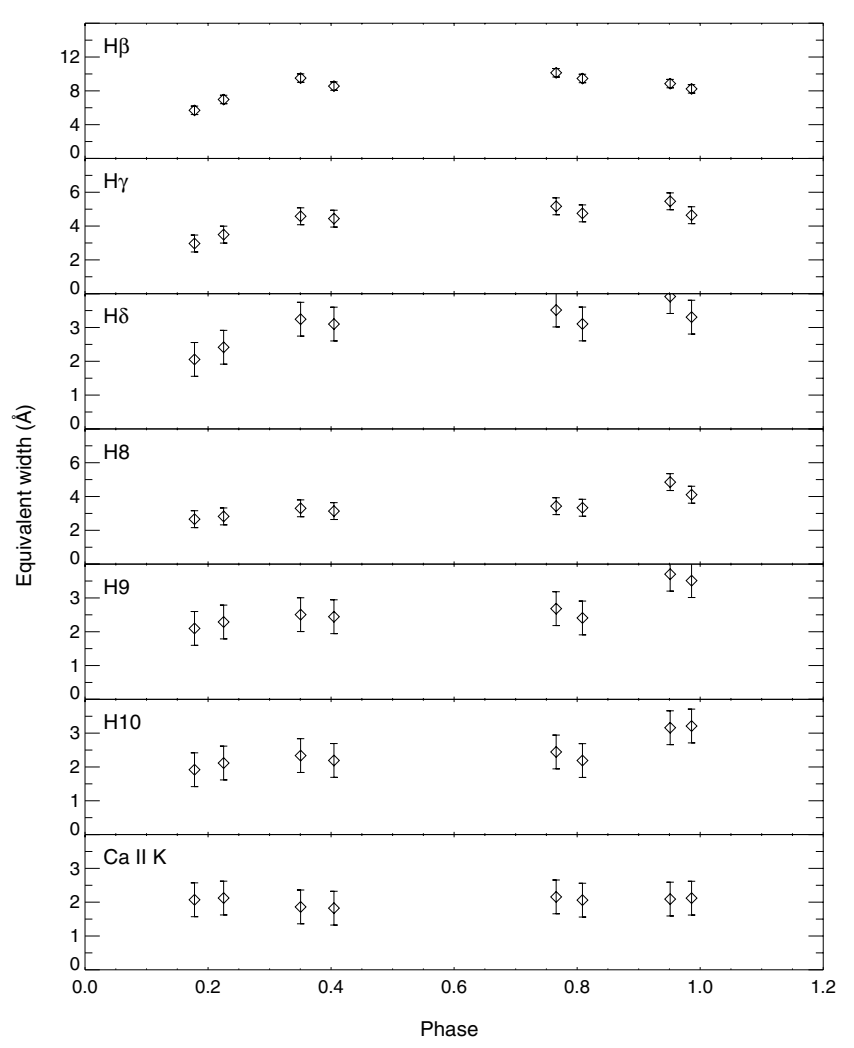

Fig. 6. The equivalent widths of the strongest emission lines in V 4046 Sgr as a function of phase.

than from the effects of shocks from colliding stellar winds or circumbinary emission, as has been suggested.

From this behavior it appears as if the various components making up the total Balmer line profiles are optically thin, and that each component only adds to the intensity at a given wavelength. This suggests that the drastic changes in the observed line shapes can be attributed to the motion of individual components around the common center of mass.

To derive restrictions on the 3-dimensional distribution of hydrogen around the components, we performed a more de-

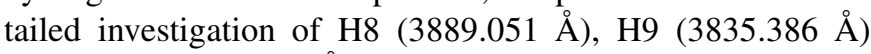
and H10 (3797.900 $)$ in particular, because the higher Balmer lines are less affected by optical depth effects than the lower Balmer lines. The three lines have a very similar shape for a given phase, and they vary with time in a similar fashion.

We have made an attempt to find a simple, common structure for the system from which the line profiles of these three lines can be calculated and compared with the observations. Careful visual inspection of the profiles of $\mathrm{H} 8, \mathrm{H} 9$ and $\mathrm{H} 10$ shows that in addition to the periodic changes in radial velocity of the two narrow components the line wings also vary periodically in velocity. This suggests the presence of two broad components with high-velocity amplitudes. At phases 0.0 and 0.5 these broad components appear at maximum velocity shifts and move to opposite sides of the line center. Thus, in the following analysis we have assumed that each $\mathrm{H}$ line is the combination of two narrow components coupled to the stars, and two broad high-velocity components.
Table 3. Parameters of the Gaussian components derived from analysis of Balmer line profiles. The peak intensities of the Gaussian components are given relative to the level of the local observed continuum. The relative intensities of the "broad" components ( 3 and 4) are different at different phases.

\begin{tabular}{lrrr}
\hline \hline Component & $\begin{array}{r}\text { Amplitude } \\
\left(\mathrm{km} \mathrm{s}^{-1}\right)\end{array}$ & Intensity & $\begin{array}{r}F W H M \\
\left(\mathrm{~km} \mathrm{~s}^{-1}\right)\end{array}$ \\
\hline 1 & 54.16 & 0.375 & 43.8 \\
2 & -56.61 & 0.30 & 43.8 \\
3 & 80.00 & (var.) & 175.0 \\
4 & -80.00 & (var.) & 164.0 \\
\hline
\end{tabular}

\subsection{Decomposition into four components}

To identify the stellar and non-stellar components of these emission lines, we numerically decomposed the combined spectrum into two stellar spectra by inverting Eq. (2). Decomposition (sometimes also called tomographic separation) of composite spectra is a standard technique for the analysis of spectroscopic binaries (see for example Bagnuolo \& Gies 1991). This method is an inverse technique. Such techniques have also been used in Doppler imaging of stellar surface structures. The main advantage of using an inverse technique is that it does not need an a priori assumption of the shape of the underlying stellar spectra, which may be difficult to construct in the case of CTTSs.

From this analysis, we confirm the existence of two narrow emission components. The components are very similar in shape and central velocity to the Ca II K profiles, only slightly broader. Also the ratio of peak intensities of these two components resembles that of the $\mathrm{Ca}$ II $\mathrm{K}$ lines. In addition, we find that the $E W$ of these narrow stellar components is identical for all phases. The relative intensities we recovered for the central components of $\mathrm{H} 8, \mathrm{H} 9$ and $\mathrm{H} 10$ scale with the oscillator strength of the respective transition, which strongly indicates that these lines indeed are formed under optically thin conditions.

After iteration for the best fit to the observed central profiles, we obtained the underlying, residual broad wing component by subtracting the narrow components from the observed line profiles. The strength of this residual profile varies with phase, but the shape turned out to be very smooth. Therefore, the high-velocity wings can be represented by two relatively broad Gaussian-shaped emission lines with a period similar to the stellar orbital period, a constant $F W H M$ and a velocity amplitude of about $80 \mathrm{~km} \mathrm{~s}^{-1}$.

Although the observed changes in intensity of the broad components demand that we scale the intensity (but not the shape!) of the broad components when reproducing the observed line profiles as a function of time, the solution we obtained consists of only four Gaussian components with welldefined properties, making it possible to focus on the physical origin of the individual components within each spectral line. We list the properties of all four components in Table 3 .

The results of our four-component approach appears very promising; we show a comparison between the observed and reproduced profiles of $\mathrm{H} 8, \mathrm{H} 9$ and $\mathrm{H} 10$ in Fig. 7. Our 


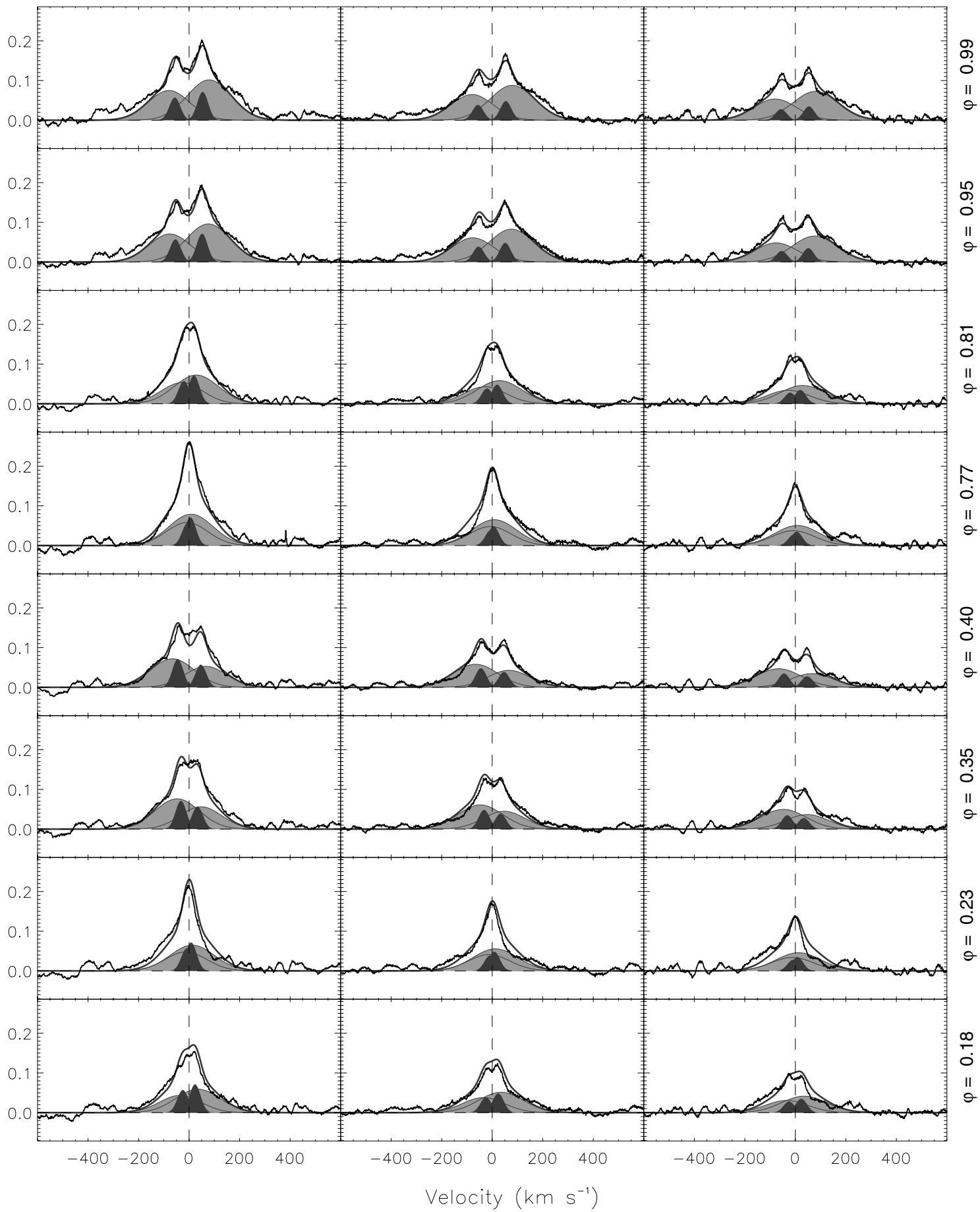

Fig. 7. A comparison between observed hydrogen line profiles (thin lines) and the reconstructed profiles (thick lines) for each observed phase of H8, H9 and H10. Synthetic spectra of the photospheric contribution have been subtracted from the observed profiles. The four Gaussian-shaped components that contribute to the reconstructed profiles are also shown. Note that the core intensities of the narrow components (dark grey) do not change with phase. The intensity scale on the vertical axis is relative to the level of the local observed continuum before subtraction of the synthetic spectra.

results show only few systematic inconsistencies. For instance, at phase 0.25 , there is a lack of redshifted emission. Occultations or aspect may play a role here. However, we could not find a clear explanation for why the $E W$ s of the broad components change, but we suspect that the rate of accretion may vary.

\section{Discussion}

The four emission line components we recovered consist of two relatively narrow components that closely follow the stellar orbit, and two relatively broad components with a period similar to the stellar period, but with a higher velocity amplitude. 
The fact that the narrow components are coupled to the stellar orbit indicates that they are formed in a region very close to each star. In addition, the close similarity in shape and behavior to $\mathrm{Ca}$ II $\mathrm{K}$ leads us to believe that the central components are formed in the extended chromosphere around each star, as discussed before in the case of Ca II K.

The broad emission line components exhibit a larger velocity amplitude than the narrow components, but with a period equal to the stellar orbital period. We did not detect any phase offset for the broad components with respect to the narrow components. We find that the simplest explanation for this behavior is to assume that there are two concentrations ("clouds") of emitting gas in the gap between the binary system and the circumstellar disk, and that these clouds co-rotate with the stars. In this configuration, the stars and the clouds are aligned, and the direction of the average velocity vector of each cloud is parallel to the velocity vector of the nearest stellar component. Then, at phase 0.0 both components have maximum velocities along the line-of-sight: blueshifted at the secondary, and redshifted at the primary.

If the clouds co-rotate with the stars, the observed velocity amplitude of $80 \mathrm{~km} \mathrm{~s}^{-1}$ of the peak intensity of these components implies that maximum emission comes from regions at $\sim 0.75\left(a_{1}+a_{2}\right)$ from the mass center. This implies that the bulk of the gas is located inside the Roche lobes of the system on each of the two sides nearest to the two Lagrangian points L2 and L3 at $\sim 1.2\left(a_{1}+a_{2}\right)$. The components in question are broad. This cannot be due solely to gas extending in the radial direction, because then the line widths would vary periodically. However, as in the models described below, one may expect that gas flows in different directions along the borders of less extensive gas clouds, which from an observational point of view would resemble broadening due to turbulence.

In recent simulations of close binaries in circular orbits with circumbinary disks Artymowicz (2003) finds that, contrary to previous calculations, substantial flows of gas from disk to stars may occur. The gas flows inwards from the disk edge and is caught by the two shocks produced on opposite sides of the mass center by the passing stars. Gas on each side is thereafter accelerated in the direction of the stellar motion and gas accumulates on each side of the binary, before continuing towards the stars, possibly forming interior disk-like structures. In Fig. 8 we show schematically how the two gas concentrations are located on each side of the binary, and we tentatively ascribe the observed variations of the broad line components to the processes described by the recent models.

We have also considered an alternative explanation where infall occurs directly from the disk to the star, but preferentially along two trajectories that are trailing each star. These flows could be located in the orbital plane, or possibly above the plane, following stellar magnetic field lines, curved both in azimuth and inclination. In this case most of the emission is formed above the shocks at the end of the infall, where gas is accelerated to velocities much higher than the stellar orbital velocities. Also, this scenario will produce maximum shifts in the radial velocities of the wing components at phases around 0.0 and 0.5 and with the proper direction of the velocity vector. However, such geometries are complex. For instance

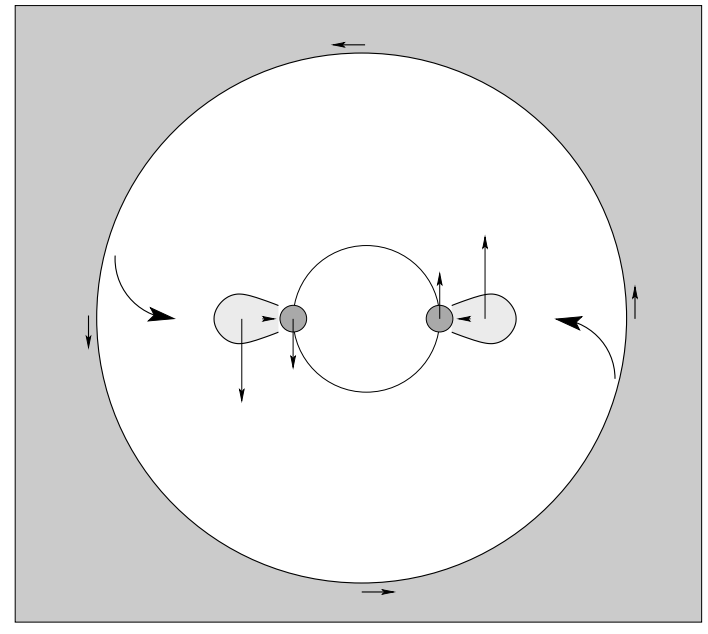

Fig. 8. This figure illustrates schematically the location of the line formation region of the "broad" components of the higher Balmer lines (H8, H9 and H10) with respect to the stars, see Sect. 6. The velocity amplitude we recovered for these components is $80 \mathrm{~km} \mathrm{~s}^{-1}$, which places these components at a distance of $0.75\left(a_{1}+a_{2}\right)$ from the center of mass, which is close to the co-linear Lagrangian points of the system. Mass transfer due to gravitational interaction between the stars and the circumbinary disk can cause matter to accelerate towards these points, where it accumulates and co-rotates with the stars, before further accretion onto the stars. We did not resolve the streamlines in our observations, but these are predicted in recent model simulations by Artymowicz (2003).

occultations of the infalling gas will occur at certain phases, and the "cone" of infall cannot be too wide or too large to maintain the wing profiles through phase. We were not able to construct a satisfactory solution for this concept.

\section{Conclusions}

We have presented an analysis of 8 high-resolution spectra $(R \approx 60000)$ of the CTTS binary V 4046 Sgr. These spectra were obtained with the UVES spectrograph at the VLT, have a high signal-to-noise ratio (up to 300) and cover a large spectral range $(3500<\lambda<6750 \AA)$. Through cross-correlation of the observed spectra we determined the orbital parameters of the binary. Although our solution is close to the ephemeris published by Quast et al. (2000), we find small but significant differences between their and our results. Thus, to obtain the best agreement with our observations, as well as with the results of Quast et al., we publish a set of revised orbital parameters. These parameters yield a more consistent mass ratio of the two components, as well as a fair agreement with published models of pre-main-sequence evolution. Our observations also indicate that both stars are in synchronous rotation.

Based on the revised orbital parameters, we calculated synthetic spectra for each star, as well as a flux-weighted combined synthetic spectrum for the binary system. These synthetic spectra allowed us to separate the photospheric absorption line spectrum from line emission, and to investigate in detail the spectral features of the system.

From the strong Li I 6707 absorption line we determined a Li abundance of $N(\mathrm{Li}) / N(\mathrm{H})=-8.5 \pm 0.2$. In our spectra 
we detected relatively low levels of veiling (from $\sim 30 \%$ of the continuum flux at $3800 \AA$ to $\sim 5 \%$ at $6200 \AA$ ). Extinction is small or absent. The strongest emission lines in V $4046 \mathrm{Sgr}$ are $\mathrm{Ca}$ II $\mathrm{H} \& \mathrm{~K}$ and the $\mathrm{H}$ lines from the Balmer series. We find small fluctuations in the EWs of the $\mathrm{H}$ lines, but not in Ca II K. However, these fluctuations cannot explain the periodic photometric variability of V $4046 \mathrm{Sgr}$ as observed by Quast et al. (2000) and Mekkaden (2000). Our time base is not long enough to detect a possible periodicity in the changes of $E W$. The Ca II $\mathrm{K}$ emission consists of two narrow components that are closely coupled to the orbit of the binary. We conclude that these lines are formed in an extended chromosphere around each star.

There is strong similarity in the shape of the Balmer lines, especially the higher members of the series. A detailed decomposition analysis of $\mathrm{H} 8, \mathrm{H} 9$ and $\mathrm{H} 10$ shows that these lines contain similar narrow components as found in $\mathrm{Ca}$ II $\mathrm{K}$. The strength of these components does not change with phase. In addition we find broad components, changing in shape with the same period as the binary, but with amplitudes of $80 \mathrm{~km} \mathrm{~s}^{-1}$, which is larger than the maximum stellar orbital velocity. This behavior suggests co-rotation with the stars, but at a distance of $0.75\left(a_{1}+a_{2}\right)$ from the center of mass, which is well within the inner edge of the circumbinary disk, and also just inside the location of the co-linear Lagrangian points of the system. Thus, substantial gas flows may exist in the gap cleared by a binary in circular motion. We also find that the $E W$ s of the broad components change with time. We do not have any clear explanation of this effect, but we suspect that accretion may vary over time.

Based on the results of our decomposition analysis of $\mathrm{H} 8$, $\mathrm{H} 9$ and $\mathrm{H} 10$ we have been able to construct one common model for these three lines. We find that the observed emission line profiles agree well with our reconstructed profiles. The structure and velocity pattern we recovered is reminiscent of recent models of accretion from a circumbinary disk to a binary system in circular motion.

Acknowledgements. Thanks go to Pawel Artymowicz for enlightening discussions, and for providing results of new binary calculations prior to publication. This work was supported by the Swedish Research Council.

\section{References}

Artymowicz, P. 2003, private communication

Artymowicz, P., \& Lubow, S. H. 1996, in Disks and Outflows Around Young Stars, ed. S. Beckwith, J. Staude, A. Quetz, \& A. Natta, Lecture Notes in Physics, 465, 115

Bagnuolo, W. G., \& Gies, D. R. 1991, ApJ, 376, 266

Baraffe, I., Chabrier, G., Allard, F., \& Hauschildt, P. H. 1998, A\&A, 337, 403

Basri, G., Johns-Krull, C. M., \& Mathieu, R. D. 1997, AJ, 114, 781
Bouvier, J., Grankin, K. N., Alencar, S. H. P., et al. 2003, A\&A, 409, 169

Busko, I. C., \& Torres, C. A. O. 1976, Informational Bulletin on Variable Stars, 1186, 1

Busko, I. C., \& Torres, C. A. O. 1978, A\&A, 64, 153

Byrne, P. B. 1986, Ir. Astron. J., 17, 294

Covino, E., Melo, C., Alcalá, J. M., et al. 2001, A\&A, 375, 130

de la Reza, R., Quast, G., Torres, C. A. O., et al. 1986, in New Insights in Astrophysics. Eight Years of UV Astronomy with IUE, ESA SP-263, 107

Duncan, D. K. 1981, ApJ, 248, 651

Gahm, G. F., Fischerström, C., Lindroos, K. P., \& Liseau, R. 1989, A\&A, 211, 115

Gahm, G. F., Petrov, P. P., Duemmler, R., Gameiro, J. F., \& Lago, M. T. V. T. 1999, A\&A, 352, L95

Gustafsson, B., Edvardsson, B., Eriksson, K., et al. 2002, in Stellar Atmosphere Modeling, ed. I. Hubeny, D. Mihalas, \& K. Werner, ASP Conf. Ser., 288

Hartmann, L. 1998, Accretion processes in star formation (Cambridge University Press)

Hilditch, R. W. 2001, An Introduction to Close Binary Stars (Cambridge University Press), 392

Jensen, E. L. N., \& Mathieu, R. D. 1997, AJ, 114, 301

Kupka, F., Piskunov, N., Ryabchikova, T. A., Stempels, H. C., \& Weiss, W. W. 1999, A\&AS, 138, 119

Mathieu, R. D. 1994, ARA\&A, 32, 465

Mathieu, R. D., Adams, F. C., \& Latham, D. W. 1991, AJ, 101, 2184

Mathieu, R. D., Ghez, A. M., Jensen, E. L. N., \& Simon, M. 2000, Protostars and Planets IV, ed. V. Mannings, A. P. Boss, \& S. S. Russell (The Univ. of Arizona Press), 703

Mathieu, R. D., Martin, E. L., \& Magazzu, A. 1996, BAAS, 28, 920

Mathieu, R. D., Stassun, K., Basri, G., et al. 1997, AJ, 113, 1841

Mekkaden, M. V. 2000, in Birth and evolution of binary stars, ed. B. Reipurth, \& H. Zinneker, Poster Proc., IAU Symp., 200, 31

Melo, C. H. F., Covino, E., Alcalá, J. M., \& Torres, G. 2001, A\&A, 378,898

Palla, F., \& Stahler, S. W. 1999, ApJ, 525, 772

Petrov, P. P., Gahm, G. F., Gameiro, J. F., et al. 2001, A\&A, 369, 993

Piskunov, N. E., Kupka, F., Ryabchikova, T. A., Weiss, W. W., \& Jeffery, C. S. 1995, A\&AS, 112, 525

Piskunov, N. E., \& Valenti, J. A. 2002, A\&A, 385, 1095

Prato, L., Simon, M., Mazeh, T., Zucker, S., \& McLean, I. S. 2002, ApJ, 579, L99

Quast, G. R., Torres, C. A. O., de la Reza, R., da Silva, L., \& Mayor, M. 2000, in Birth and evolution of binary stars, ed. B. Reipurth, \& H. Zinneker, Poster Proc., IAU Symp., 200, 28

Ryabchikova, T. A., Piskunov, N., Stempels, H. C., Kupka, F., \& Weiss, W. W. 1999, Phys. Scr, T83, 162

Silber, J., Gledhill, T., Duchêne, G., \& Ménard, F. 2000, ApJ, 536, L89

Stempels, H. C., \& Piskunov, N. 2002, A\&A, 391, 595

Stempels, H. C., \& Piskunov, N. 2003, A\&A, 408, 693

Takami, M., Bailey, J., Gledhill, T. M., Chrysostomou, A., \& Hough, J. H. 2001, MNRAS, 323, 177

Valenti, J. A., \& Piskunov, N. 1996, A\&AS, 118, 595

Zahn, J.-P. 1977, A\&A, 57, 383

Zahn, J.-P., \& Bouchet, L. 1989, A\&A, 223, 112 